\title{
Bruxisme et réponses posturales avant et après traitement occlusal
}

Pierre Louis Bernard ${ }^{1}$, Oliver Jame ${ }^{2}$, Sofiane Ramdani ${ }^{1}$, Benoît Seigle ${ }^{1}$, Francis Degache ${ }^{3}$, Philippe Collin ${ }^{1}$, Jean-Paul Micallef ${ }^{1}$, Hubert Blain ${ }^{1}$ et Isabelle Bonafé ${ }^{2}$

1 Movement to Health (M2H), Euromov. Université Montpellier 1, 700 avenue du Pic Saint-Loup, 34090 Montpellier, France

2 UFR Odontologie, 545 avenue du Pr J.L. Viala, 34193 Montpellier, France

3 Institute of Sport Sciences (ISSUL), Département de Physiologie, Faculté de Biologie et de Médecine, Université de Lausanne, Suisse

Reçu le 5 décembre 2012 - Accepté le 16 mai 2013

Résumé. Objectif - Le but de ce travail était de quantifier l'influence du bruxisme sur les réponses posturales et de définir l'influence du port d'une orthèse occlusale de relaxation neuromusculaire sur les valeurs de centre de pression plantaire (CPP). Méthode - Neuf sujets témoins et sept sujets bruxomanes composaient la population soumise aux tests stabilométriques les yeux ouverts (YO) et les yeux fermés (YF) sur sol dur, en PIM (Position d'Intercuspidie Maximale), en PR (Position de Repos) et POgt (Position d'Occlusion avec gouttière). Résultats - Les principaux résultats ont révélé une absence d'influence significative du bruxisme sur les valeurs de CPP mesurées les yeux ouverts et les yeux fermés, sujets en PIM et sujet en PR. Pour ce qui est de l'influence du port d'une orthèse occlusale, nous observons une absence systématique de différences significatives entre la position d'intercuspidie maximale (PIM) et la position d'occlusion avec gouttière (POgt). Après 15 jours de port de l'orthèse, nous n'observons pas de différence manifeste, tandis que 30 jours de port entraînent des différences significatives en position de repos, les yeux ouverts, pour la position médio-latérale $(p<0,01)$, et en position d'intercupidie maximale pour la position antéro-postérieure, les yeux fermés $(p<0,02)$.

Mots clés : Posture, bruxisme, stabilométrie, orthèse occlusale

\begin{abstract}
Bruxism disorder and postural stability before and after occlusal splint treatment.

Objective - We aimed at examining the effect of bruxism on postural responses on specific balance regulation and the influence of occlusal splint on mid-term and long-term postural responses. Method - The balance stability of nine healthy subjects and seven patients presenting bruxism was assessed using a force platform with and without vision on a firm floor. We analysed stabilometric data for surface, length, antero-posterior and medio-lateral projection of the center of pressure (COP) sway. During this static postural evaluation, subjects were tested in rest occlusal position, vertical occlusal position and occlusal position with orthesis. Results - The analysis showed no significant effect of bruxism on COP values in the test with $\mathrm{OE}$ and $\mathrm{CE}$ in rest occlusal position and vertical occlusal position. For the influence of occlusal splint treatment, we observed no significant difference of responses between the vertical experimental occlusal position and the vertical occlusal position with splint. Moreover, no significant difference was observed after 15 days of occlusal splint treatment and only two differences after 30 days $(0.01<p<0.02)$.
\end{abstract}

Key words: Posture, bruxism, stabilometry, occlusal splint

\section{Introduction}

Depuis des années, le rôle et les effets de l'occlusion dentaire sur le système postural suscitent un intérêt croissant. De nombreux auteurs ont essayé d'approfondir le sujet de façon à clarifier les corrélations existantes (Gangloff, Louis, \& Perrin, 2000; Milani, De Perière, Lapeyre, \& Pourreyron, 2000; Perinetti, 2006). L'influence de la posture sur l'occlusion (Mc Lean, Brennan, \& Friedman, 1973 ; Preiskel, 1965 ; Serviere, 1989; Tripodakis, Smulow, 
Mehta, \& Clark, 1995), et, à l'inverse, l'influence de l'occlusion sur la posture (Kibana, Ishijima \& Hirai, 2002; Urbanowicz, 1991) constituent deux thèmes qui justifient divers travaux. Une partie de ces réflexions s'est développée dans le but d'améliorer les performances sportives et a débouché sur la création de dispositifs orthopédiques ou d'orthèses occlusales. Quelques études (Laplanche, 1992 ; Stenger, Ricketts, Lawton, \& Wright, 1978) concluent à une amélioration des performances sportives par le port d'une orthèse occlusale sans indiquer pourquoi et comment ce dispositif peut influer sur l'organisme. À l'inverse, d'autres études (Bricot \& Gahery; Greenberg, Cohen, Spinger, Kotwick, \& Vergo, 1981; Vergo, Kotwick, Cohen, \& Greenberg, 1981) ne révèlent aucune influence significative du port d'une orthèse sur la performance.

Des dysfonctionnements de l'appareil manducateur et plus spécialement le bruxisme, peuvent générer des tensions au niveau des muscles masticateurs et les transmettre par relais aux muscles des régions cervicale et nucale, responsables de la posture de la tête. Chez différentes populations, le rôle de l'orientation et de la stabilisation de la tête ont été mis en évidence lors de l'étude des mécanismes du contrôle de la posture (De Nunzio, Nardone, \& Schiappati 2005; Gresty, 1987; Norré, 1995; Tardieu, Dimitrescu, Giraudeau, Blanc, Cheynet, \& Borel, 2009).

Le bruxisme est une pathologie caractérisée par des « contractions involontaires et inconscientes des muscles masticateurs dentaires généralement avec contacts dentaires » (CNO, 2001). Le résultat de ces contractions inconscientes et non fonctionnelles, diurnes ou nocturnes (ou les deux), se traduit par le serrement, forme statique (bruxisme centré) et/ou le grincement ou le claquement des dents, forme dynamique (bruxisme excentré). Le diagnostic sera réalisé lors d'un examen endo-buccal, qui descellera la présence de fêlures, de fractures, de facettes d'usure plus ou moins généralisées à toute la dentition, mais également lors d'un examen exo- buccal avec palpation des muscles masséters et temporaux, sterno-cléidomastoïdiens et trapèzes. Un examen complémentaire basé sur l'analyse électromyographique des muscles masticateurs pourra confirmer ce diagnostic (Clark, Rugh \& Handelman, 1980; Solberg, Clark \& Rugh, 1975). La prise en charge mécanique de cette pathologie s'effectue par orthèse de relaxation neuromusculaire dont le but principal est de réduire l'hyperactivité musculaire en augmentant la stabilité occlusale.

L'objectif essentiel de ce travail est de définir l'influence du bruxisme sur les réponses posturales par l'intermédiaire de trois démarches. La première sert l'analyse de l'influence du bruxisme en comparant les sujets sains aux sujets bruxomanes. La seconde vise à définir l'influence immédiate du port d'une orthèse occlusale lors de test en situation de position d'intercuspidie maximale (PIM) et en position d'occlusion avec gouttière (POgt). La dernière démarche étudie l'influence du port
Tableau 1. Valeurs moyennes d'âge, de taille et de masse des deux groupes de sujets.

\begin{tabular}{cccc}
\hline & Age $($ ans $)$ & Taille $(\mathrm{cm})$ & Masse $(\mathrm{kg})$ \\
\hline Sain & 25,4 & 174,7 & 77,7 \\
Bruxomane & 32,4 & 172 & 66,8 \\
\hline
\end{tabular}

d'une orthèse à court et à moyen terme en comparant les évolutions des deux groupes sain et bruxomane, à 15 et à 30 jours.

\section{Méthodologie}

\subsection{Population}

Les sujets ont été inclus, de manière prospective au centre de soins et de consultations dentaires (centre hospitalier universitaire de Montpellier) dans une étude, non invasive, d'évaluation diagnostique (Tab. 1).

Ils ont été recrutés lors d'une consultation hebdomadaire réservée aux dysfonctions crânio-mandibulaires et testés par un seul examinateur chirurgien dentiste lors d'un examen clinique comprenant un questionnaire, un examen endo-buccal, exo-buccal, articulaire et musculaire et après recueil du consentement éclairé.

Les sept sujets pathologiques présentaient une symptomatologie clinique évocatrice de bruxisme sans pathologie articulaire, répondant ainsi à des critères diagnostiques, dentaires, musculaires, qui permettaient de les catégoriser (classe d'angle, schéma occlusal, absence de dents non remplacées, présence de prothèses dentaires [fixe ou amovible], fractures dentaires, caries dentaires, pathologies gingivales et/ou parodontales).

Un groupe de neuf sujets adultes asymptomatiques, ne présentant aucune pathologie d'ordre fonctionnel a été sélectionné en raison de la normalité de l'examen clinique musculaire et articulaire. Des examens d'imagerie (IRM) ont été réalisés dans le service de neuroradiologie du CHU de Montpellier selon un protocole standardisé, et ont confirmé l'absence de pathologie articulaire.

Ont été exclus les sujets présentant une pathologie de l'appareil manducateur autre que le bruxisme (traumatismes articulaires ou neurologiques sévères). Les troubles neurologiques, psychologiques et locomoteurs susceptibles d'influencer les réponses posturales ainsi que l'incapacité à revenir aux dates programmées constituaient également des critères de non-inclusion.

\subsection{Protocole}

Deux groupes de sujets, sains et bruxomanes, sont étudiés dans diverses situations expérimentales, les yeux ouverts (YO) et les yeux fermés (YF), bouche fermée en PIM c'est-à-dire en position dentaire caractérisée par le maximum de contacts, bouche entrouverte en position de 
repos (PR) et bouche fermée en POgt. Ces gouttières sont dites de relaxation neuromusculaire et ont pour objectif principal, la perte de la mémoire occlusale en empêchant l'établissement de l'intercuspidation existante (Rey, Chossegros, El Zoghby, Carlier, Perez, \& Orthlieb, 2009, Unger \& Brocard, 1996). Elles modifient et redistribuent l'influx sensoriel et tactile des dents, en augmentant légèrement la dimension verticale d'occlusion, ce qui aboutit à la disparition progressive des contractions musculaires (Rozenweig, 1994).

\subsubsection{Conception et réalisation de l'orthèse}

Ces gouttières ou orthèses sont des appareils en résine acrylique dure, lisse, transparente, qui couvrent la surface occlusale de toute l'arcade mandibulaire, ainsi que le tiers des faces vestibulaires.

Leur réalisation doit répondre à un cahier des charges très précis et selon un protocole standard. Une prise d'empreinte des maxillaires supérieur et inférieur est réalisée avec un matériau d'enregistrement spécifique nommé l'alginate (hydrocolloïde irréversible), le patient est en position assise avec la tête droite.

Le choix du porte empreinte est fonction de la largueur et de la longueur de l'arcade (la totalité des dents doit être recouverte) et une absence d'interférences avec un élément anatomique (palais, trigone rétro molaire, face occlusale, face vestibulaire ou linguale) est impérativement requise. La prise d'un arc facial permet d'enregistrer la position du maxillaire supérieur par rapport à la base du crâne tandis qu'une cire de PIM situe la mandibule par rapport au maxillaire. Les empreintes, coulées en plâtre dur, sont alors montées sur simulateur (SAM) grâce à ces deux enregistrements. Les modèles ainsi positionnés reproduisent l'engrènement dentaire du patient et permettent de visualiser les mouvements d'ouverture et de fermeture buccale (Fig. 1).

Cette orthèse devra être stable, rétentive, peu encombrante. Sa surface occlusale doit être plane et répondre à des principes clairement établis (Figs. 2a, 2b) :

- elle doit être uniformément lisse, sans indentation;

- elle doit établir, en PIM, des contacts simultanés avec toutes les cuspides supports antagonistes;

- elle doit guider les déplacements mandibulaires en respectant un schéma occlusal idéal (latéralité avec guidage canin et absence de contact postérieur, propulsion avec guidage incisif et désocclusion postérieure). Cette surface occlusale doit être adaptée aux changements qui interviennent dans les rapports intermaxillaires par modification de l'activité musculaire, et une vérification régulière sera réalisée par le praticien à 15 jours, un mois puis à trois mois et six mois.

\subsubsection{Protocole d'évaluation stabilométrique}

Les évaluations se sont déroulées dans la faculté d'odontologie de Montpellier. Celles-ci répondaient aux normes

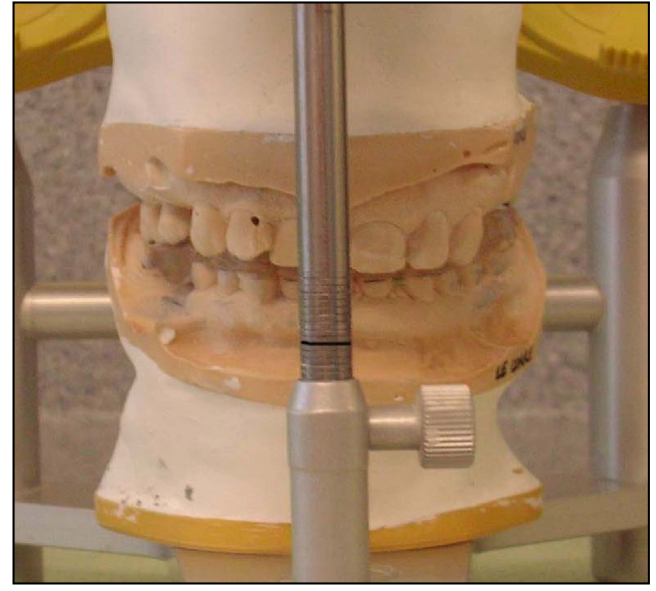

Fig. 1. Orthèse de relaxation neuromusculaire.

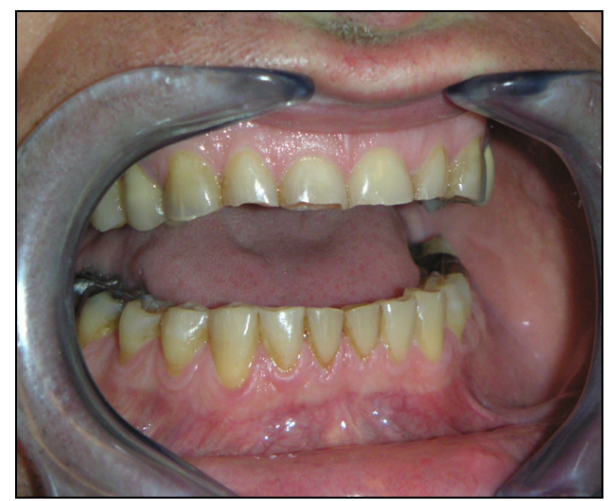

(a)

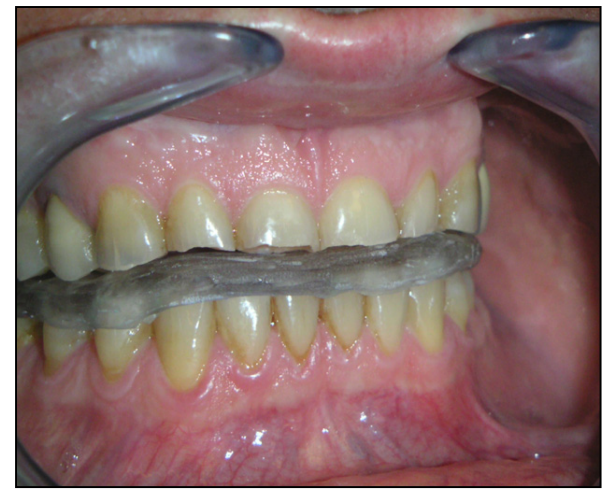

(b)

Fig. 2. (a) \& (b). Vue clinique avant et après la mise en place de l'orthèse.

de passation des épreuves posturologiques concernant les dimensions, la luminosité et le bruit environnemental. Les épreuves se déroulaient dans le respect des normes d'évaluation des épreuves statiques les YO et les YF sur sol dur (American Academy of Neurology, 1993). Chacun des groupes était évalué en condition les YO puis les YF. Au sein de ces deux conditions visuelles, un tirage 
au sort aléatoire déterminait l'ordre de passage entre les trois situations de PR, de PIM et de POgt.

La PR correspond à l'absence de contact entre l'arcade maxillaire et l'arcade mandibulaire. La POgt imposait aux personnes du groupe expérimental, le port d'une orthèse placée au niveau du maxillaire inférieur. Pour ces derniers, les acquisitions étaient effectuées en PR, PIM et POgt suite à un tirage au sort aléatoire. Pour le groupe témoin, les acquisitions étaient effectuées après tirage au sort en PR et en PIM. L'évaluation initiale débutait à J0. Une procédure identique était reproduite à quinze jours $(\mathrm{J} 15)$ puis à 30 jours $(\mathrm{J} 30)$. Suite à l'évaluation initiale, le groupe expérimental bruxomane portait quotidiennement de manière nocturne, son orthèse.

Lors de l'évaluation stabilométrique, les participants devaient se maintenir en station verticale immobile, les YO et les YF sur une plaque de mesure Médicapteurs Win Posturo $40 \mathrm{~Hz} / 16 \mathrm{~b}{ }^{\circledR}$ (Toulouse, France) durant un temps d'acquisition de 25,6 secondes. Les participants étaient en chaussettes, les pieds orientés chacun de $15^{\circ}$ latéralement par rapport au plan sagittal, avec les talons distants l'un de l'autre, de quatre centimètres. Les bras étaient ballants le long du corps avec la tête droite et le regard fixait un repère visuel à hauteur du regard, situé à un mètre. Ces conditions étaient respectées tout au long de l'acquisition ainsi qu'en condition de privation visuelle suite à un ancrage initial du regard. Avant le premier enregistrement, une période de familiarisation sur sol dur en condition les YO était imposée aux personnes pour une durée proche de $25 \mathrm{~s}$. À l'issue de cette familiarisation, les participants disposaient de $30 \mathrm{~s}$ de récupération avant la première des acquisitions. Un seul essai était réalisé par condition expérimentale.

Le système d'analyse des activités posturo-cinétiques Medicapteurs $40 \mathrm{~Hz} / 16 \mathrm{~b}{ }^{\circledR}$ permettait l'acquisition des signaux. Munis de capteurs de force à jauge de déformation, la capacité de charge de chacun des trois capteurs était de plus de $100 \mathrm{~kg}$. La plaque mesurait les réactions au sol, des forces verticales $(\mathrm{Fz})$ exercées sur les axes médiolatéral $(-\mathrm{X}, \mathrm{X})$ et antéropostérieur $(-\mathrm{Y}, \mathrm{Y})$. L'acquisition du signal s'effectuait à une fréquence de $40 \mathrm{~Hz}$. Un convertisseur analogique digital sur 16 bits amplifiait et linéarisait le signal. Un filtre passe bas permettait le passage du signal en éliminant ses bruits à une fréquence de coupure de $0,4 \mathrm{~Hz}$. Il était relié à une unité centrale informatique portable équipée du logiciel Medicapteurs Winposture $2000 \mathrm{~V}-2.24 .12^{\circledR}$ qui calculait les positions successives du barycentre des pressions plantaires exercées par le sujet, sur le plateau, pendant la durée d'échantillonnage.

Les déplacements du centre de pression plantaire (CPP) enregistrés permettaient l'analyse des paramètres de surface occupée par le trajet du CPP $\left(\right.$ en $\left.\mathrm{mm}^{2}\right)$, de la longueur du trajet parcouru par le CPP (en mm), de la position du CPP dans les axes médio-latéral (X en mm) et antéro-postérieur (Y en $\mathrm{mm}$ ).

\subsubsection{Analyse statistique}

La normalité des distributions des données stabilométriques a été initialement traitée par le test de Kolmogorov-Smirnov. Pour des raisons d'absence de normalité de la distribution et taille des échantillons, nous avons systématiquement retenu des statistiques nonparamétriques. Aussi, dans le traitement des effets à court et à moyen terme, l'incapacité à traiter des interactions entre les groupes (sain vs bruxomane) et le temps (J0 vs J15 vs J30), nous a incités à une démarche plus analytique utilisant les différences entre les temps J0 vs J15 et J0 vs J30 pour les deux groupes indépendants.

Le test de Mann-Whitney a permis de traiter la première hypothèse et de comparer les deux groupes indépendants sain versus bruxomane en PIM et en PR en conditions les $\mathrm{YO}$ et les $\mathrm{YF}$. Ce test a été à nouveau utilisé pour déterminer l'effet à court terme (J0 vs J15) et à moyen terme ( 0 vs J30) du port d'une gouttière par la comparaison de l'évolution des valeurs stabilométriques entre les deux groupes en PIM et en PR, en conditions les YO et les YF. Le test de Wilcoxon a permis de déterminer l'effet immédiat du port d'une gouttière et de comparer les valeurs du groupe expérimental de bruxomanes à J0 en PIM versus les YO et les YF en PR.

\section{Résultats}

\subsection{Influence du bruxisme : sain vs bruxomane}

À l'exception de la projection du CPP dans l'axe antéropostérieur en condition les YF en PR $(p<0,04)$ (Fig. 4), l'observation des résultats montre une absence systématique de différence manifeste entre le groupe sain et le groupe bruxomane pour tous les paramètres stabilométriques mesurés les YO et les YF, en condition PIM et PR (Fig. 3, Fig. 4).

\subsection{Influence immédiate du port d'une orthèse occlusale : PIM vs POgt}

La comparaison des valeurs stabilométriques mesurées chez le groupe bruxomane les YO et les YF montre une absence systématique de différence significative entre la PIM et la POgt (Fig. 5).

\subsection{Influence du port d'une orthèse à court et à moyen termes : comparaison des évolutions des deux groupes sain et bruxomane entre $\mathrm{JO}$ et $\mathrm{J} 15$ (court terme) et entre $\mathrm{J} 0$ et $\mathrm{J} 30$ (moyen terme)}

L'observation des valeurs d'évolution des paramètres stabilométriques suite à 15 jours de port de l'orthèse, chez le groupe bruxomane, montre une absence de différence 

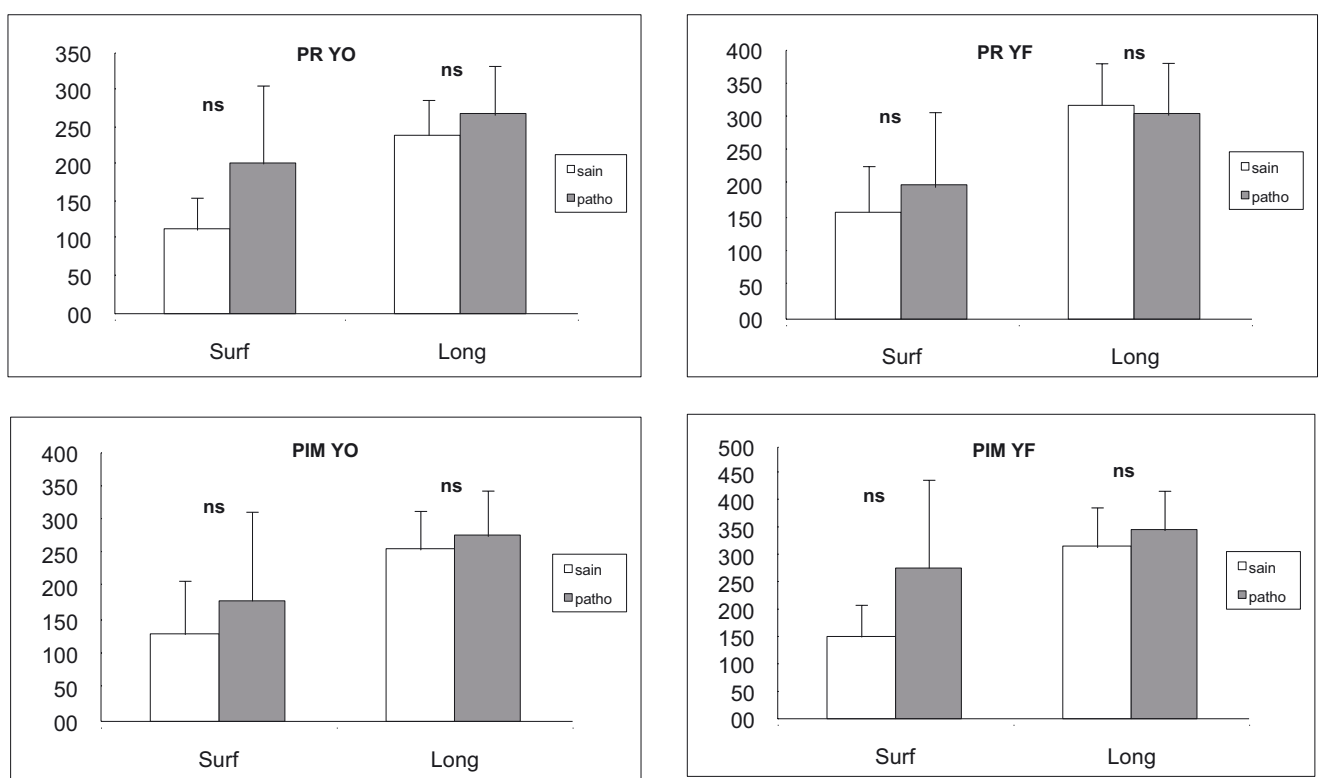

Fig. 3. Valeurs moyennes et écart types des deux groupes sain et pathologique testés les yeux ouverts (YO) et les yeux fermés $(\mathrm{YF})$, en position de repos (PR) et en position d'intercuspidation maximale (PIM) pour les paramètres stabilométriques du centre de pression plantaire (CPP) de surface $\left(\mathrm{mm}^{2}\right)$ et de longueur $(\mathrm{mm})$.
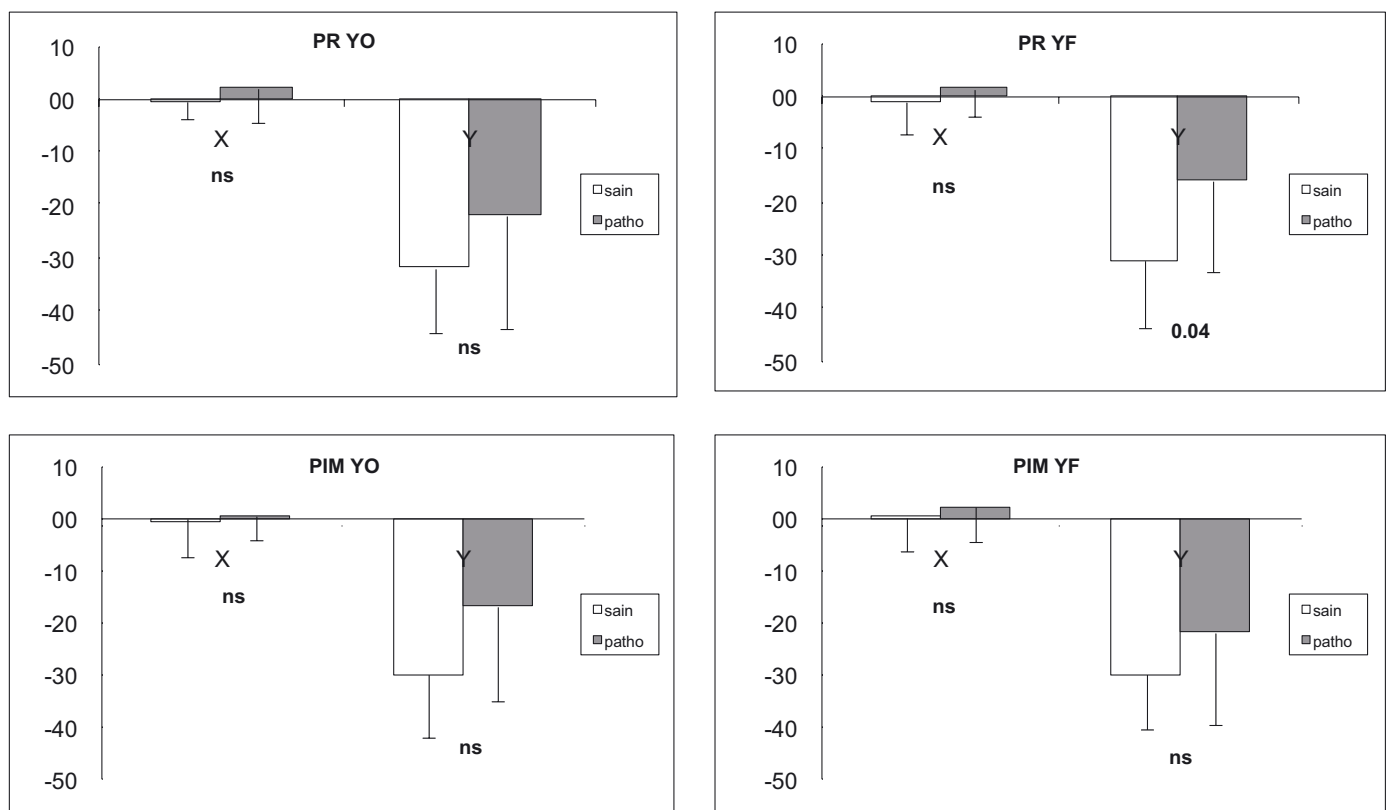

Fig. 4. Valeurs moyennes et écart types des deux groupes sain et pathologique testés les yeux ouverts (YO) et les yeux fermés $(\mathrm{YF})$, en position de repos (PR) et en position d'intercuspidation maximale (PIM) pour les paramètres stabilométriques de centre de pression plantaire (CPP) dans le plan médio-latéral (X en mm) et antéro-postérieur (Y en $\mathrm{mm}$ ).

caractéristique tant en PR qu'en PIM (Tab. 2a). Pour ce qui concerne l'influence du port de l'orthèse sur une durée de 30 jours (Tab. 2b), la comparaison des évolutions des paramètres stabilométriques montre des différences significatives en PR, les YO, pour la position médio-latérale $(p<0,01)$ et en PIM pour la position antéro-postérieure, les YF, $(p<0,02)$.

\section{Discussion}

La première question qui a guidé notre travail concerne l'influence du bruxisme sur les réponses posturales. Nous émettions l'hypothèse d'une l'influence significative de l'activité de bruxisme depuis plusieurs années, sur les réponses posturales. La comparaison des deux groupes 

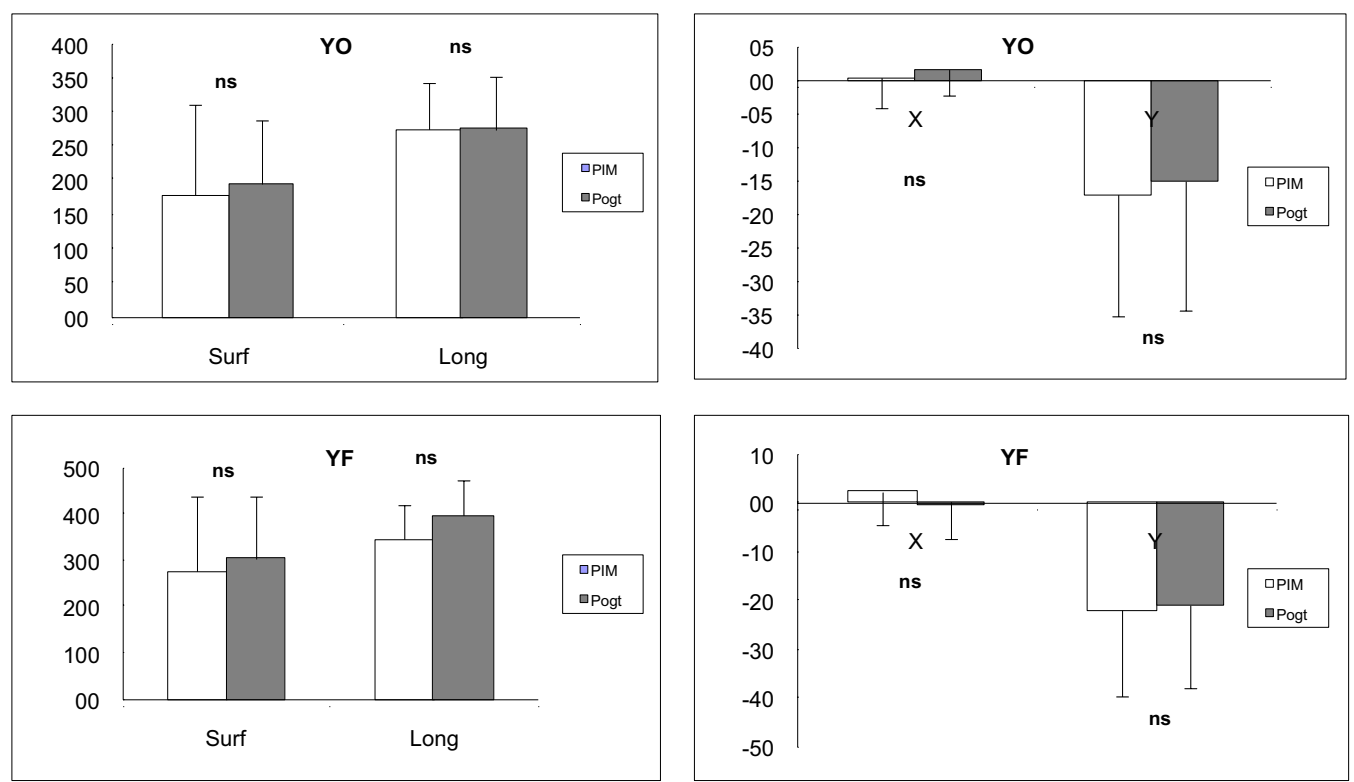

Fig. 5. Valeurs moyennes (m) et écart types (sd) du groupe pathologique testé les yeux ouverts (YO) et les yeux fermés (YF), en position d'intercuspidation maximale (PIM) et en position d'occlusion avec gouttière (POgt) pour les paramètres de surface $\left(\mathrm{mm}^{2}\right)$, de longueur $(\mathrm{mm})$ et de position médio-latérale (X en $\mathrm{mm}$ ) et antéro-postérieure ( $\mathrm{Y}$ en $\mathrm{mm}$ ) du centre de pression plantaire.

Tableau 2. (a) \& (b) Valeurs moyennes (m) et écart types (sd) de la différence de valeurs des évaluations à J0 versus J15 (a) et à J0 versus J30 (b) pour les deux groupes sain (Sa) et pathologique (Pa), testés les yeux ouverts (YO) et les yeux fermés $(\mathrm{YF})$, en position de repos (PR) et en position d'intercuspidation maximale (PIM).

\begin{tabular}{|c|c|c|c|c|c|c|c|c|c|}
\hline \multicolumn{10}{|c|}{ YO JO / J15 } \\
\hline & & \multicolumn{4}{|c|}{ PR } & \multicolumn{4}{|c|}{ PIM } \\
\hline & & $\begin{array}{c}\mathbf{S f} \\
\mathrm{mm}^{2}\end{array}$ & $\begin{array}{l}\mathrm{Lg} \\
\mathrm{mm}\end{array}$ & $\begin{array}{c}\mathbf{X} \\
\mathrm{mm}\end{array}$ & $\begin{array}{c}\mathbf{Y} \\
\mathrm{mm}\end{array}$ & $\begin{array}{c}\mathbf{S f} \\
\mathrm{mm}^{2}\end{array}$ & $\begin{array}{l}\mathrm{Lg} \\
\mathrm{mm}\end{array}$ & $\begin{array}{c}\mathbf{X} \\
\mathrm{mm}\end{array}$ & $\begin{array}{c}\mathbf{Y} \\
\mathrm{mm}\end{array}$ \\
\hline \multirow[t]{2}{*}{$\mathrm{Sa}$} & $\mathrm{m}$ & $-24,8$ & $-27,1$ & 3,7 & $-3,1$ & $-6,9$ & $-5,4$ & 0,9 & $-1,7$ \\
\hline & sd & 58,1 & 70,3 & 5,1 & 11,7 & 40,6 & 42,4 & 8,5 & 10,2 \\
\hline \multirow[t]{4}{*}{$\mathrm{Pa}$} & $\mathrm{m}$ & 35,7 & $-13,9$ & 2,2 & $-7,3$ & $-6,3$ & $-1,3$ & 0,0 & $-1,5$ \\
\hline & sd & 54,4 & 52,5 & 4,2 & 20,2 & 118,6 & 75,7 & 3,9 & 15,0 \\
\hline & $z$ & $-1,85$ & $-0,26$ & 0,68 & 0,58 & 0,26 & $-0,63$ & 0,58 & 0,15 \\
\hline & $p<$ & NS & NS & NS & NS & NS & NS & NS & NS \\
\hline
\end{tabular}

\begin{tabular}{cccccccc}
\hline \multicolumn{9}{c}{ PR } & \multicolumn{7}{c}{ PIM JO / J15 } \\
\hline $\begin{array}{c}\text { Sf } \\
\mathrm{mm}^{2}\end{array}$ & $\mathbf{L g}$ & $\mathbf{X}$ & $\mathbf{Y}$ & $\mathbf{S f}$ & $\mathbf{L g}$ & $\mathbf{X}$ & $\mathbf{Y}$ \\
$\mathrm{mm}$ & $\mathrm{mm}$ & $\mathrm{mm}^{2}$ & $\mathrm{~mm}$ & $\mathrm{~mm}$ & $\mathrm{~mm}$ \\
\hline$-11,9$ & $-33,4$ & 0,0 & $-2,8$ & $-16,2$ & 5,9 & 0,0 & $-4,0$ \\
107,6 & 59,4 & 10,7 & 9,4 & 86,6 & 46,2 & 8,6 & 7,6 \\
\hline$-11,6$ & $-68,1$ & 0,9 & $-1,6$ & 12,9 & $-33,7$ & 1,5 & $-5,8$ \\
55,4 & 106,2 & 5,2 & 9,0 & 135,6 & 92,7 & 5,2 & 11,1 \\
\hline $\mathbf{0 , 3 7}$ & $\mathbf{0 , 4 7}$ & $\mathbf{0 , 1 5}$ & $\mathbf{- 0 , 7 9}$ & $-\mathbf{0 , 5 8}$ & $\mathbf{0 , 4 7}$ & $\mathbf{- 0 , 2 6}$ & $\mathbf{0 , 6 9}$ \\
$\mathbf{N S}$ & $\mathbf{N S}$ & $\mathbf{N S}$ & $\mathbf{N S}$ & $\mathbf{N S}$ & $\mathbf{N S}$ & $\mathbf{N S}$ & $\mathbf{N S}$ \\
\hline
\end{tabular}

\begin{tabular}{|c|c|c|c|c|c|c|c|c|c|}
\hline \multicolumn{10}{|c|}{ YO JO / J30 } \\
\hline & & \multicolumn{4}{|c|}{ PR } & \multicolumn{4}{|c|}{ PIM } \\
\hline & & $\begin{array}{c}\mathbf{S f} \\
\mathrm{mm}^{2}\end{array}$ & $\begin{array}{r}\mathrm{Lg} \\
\mathrm{mm}\end{array}$ & $\begin{array}{c}\mathbf{X} \\
\mathrm{mm}\end{array}$ & $\begin{array}{c}\mathbf{Y} \\
\mathrm{mm}\end{array}$ & $\begin{array}{c}\mathbf{S f} \\
\mathrm{mm}^{2}\end{array}$ & $\begin{array}{c}\mathrm{Lg} \\
\mathrm{mm}\end{array}$ & $\begin{array}{c}\mathbf{X} \\
\mathrm{mm}\end{array}$ & $\begin{array}{c}\mathbf{Y} \\
\mathrm{mm}\end{array}$ \\
\hline \multirow[t]{2}{*}{ Sa } & $\mathrm{m}$ & 21,7 & $-2,4$ & $-1,2$ & 6,0 & 25,3 & 14,6 & $-0,4$ & 7,4 \\
\hline & sd & 24,4 & 72,7 & 6,7 & 7,6 & 89,4 & 66,4 & 9,3 & 10,5 \\
\hline \multirow[t]{4}{*}{$\mathrm{Pa}$} & $\mathbf{m}$ & 40,9 & 1,7 & 8,3 & $-2,7$ & 21,9 & 1,0 & 5,8 & 4,0 \\
\hline & sd & 145,3 & 66,9 & 5,4 & 13,4 & 112,0 & 74,1 & 6,1 & 14,8 \\
\hline & $\mathbf{z}$ & 0,37 & $-0,10$ & $-2,59$ & 1,32 & 0,37 & 0,47 & $-1,53$ & 0,89 \\
\hline & $p<$ & NS & NS & 0,01 & NS & NS & NS & NS & NS \\
\hline
\end{tabular}

\begin{tabular}{|c|c|c|c|c|c|c|c|}
\hline \multicolumn{8}{|c|}{ YF JO / J30 } \\
\hline \multicolumn{4}{|c|}{ PR } & \multicolumn{4}{|c|}{ PIM } \\
\hline $\begin{array}{c}\mathbf{S f} \\
\mathrm{mm}^{2}\end{array}$ & $\begin{array}{l}\mathrm{Lg} \\
\mathrm{mm}\end{array}$ & $\begin{array}{c}\mathbf{X} \\
\mathrm{mm}\end{array}$ & $\begin{array}{c}\mathrm{Y} \\
\mathrm{mm}\end{array}$ & $\begin{array}{c}\mathbf{S f} \\
\mathrm{mm}^{2}\end{array}$ & $\begin{array}{l}\mathrm{Lg} \\
\mathrm{mm}\end{array}$ & $\begin{array}{c}\mathbf{X} \\
\mathrm{mm}\end{array}$ & $\begin{array}{c}\mathrm{Y} \\
\mathrm{mm}\end{array}$ \\
\hline$-60,8$ & $-29,1$ & $-0,8$ & 3,7 & $-36,4$ & $-62,6$ & 1,7 & 5,9 \\
\hline 96,9 & 85,8 & 9,3 & 12,1 & 49,0 & 110,4 & 6,9 & 6,4 \\
\hline$-24,3$ & $-51,9$ & 4,0 & 7,1 & 47,7 & 18,4 & 3,8 & $-5,5$ \\
\hline 61,1 & 101,0 & 8,9 & 10,5 & 132,5 & 62,0 & 9,3 & 10,0 \\
\hline$-0,79$ & 0,58 & $-1,37$ & $-0,47$ & $-1,32$ & $-1,74$ & $-0,68$ & 2,54 \\
\hline NS & NS & NS & NS & NS & NS & NS & 0,02 \\
\hline
\end{tabular}

expérimentaux bruxomane et témoin sain en conditions les YO et les YF, en PR et en PIM, avant toute prise en charge par l'orthèse, montre une absence systématique de différence significative entre les deux groupes. Il s'avère néanmoins possible d'évoquer quelques différences de comportements entre les deux groupes. Ainsi, les paramètres de surface et de $\mathrm{Y}$ présentent des valeurs moyennes qui différencient les deux groupes; en condition PR pour la modalité, les YO, les deux groupes ont respectivement $112 \mathrm{~mm}^{2}( \pm 43)$ vs $201 \mathrm{~mm}^{2}( \pm 104,9)$ au paramètre surface (Fig. 3) tandis que pour le paramètre Y (Fig. 4), les valeurs des deux groupes sont respectivement de $-30,3 \mathrm{~mm}( \pm 12,1)$ vs $-17,1 \mathrm{~mm}( \pm 18,2)$ en condition PIM pour la modalité YO et de $-31,4 \mathrm{~mm}$ 
$( \pm 12,7)$ vs $-16.4 \mathrm{~mm}( \pm 17,2)$ en condition $\mathrm{PR}$ pour la modalité YF. La petite taille et l'hétérogénéité des capacités de régulation posturale de l'échantillon masquet-elle une tendance des bruxomanes à avoir une moins grande stabilité et à se projeter plus en avant pour assurer leur équilibration? Il est considéré que le fait de serrer les dents (clunching) provoque le déplacement du massif céphalique vers l'avant (Hartmann \& Cucchi, 1993). Le rôle d'une position plus antérieure sur la mise en tension des muscles de la chaine postérieure (gastrocnémius, soléaire, ischio-jambiers) favorisant des tensions excessives et une moindre capacité à réguler la posture, de part une fatigabilité neuro-musculaire locale, constituent des pistes de travail que nous envisageons de réaliser.

Le deuxième questionnement porte sur l'influence de l'augmentation de la dimension verticale par le port d'une orthèse occlusale. Nos résultats ne révèlent aucune différence significative de réponses posturales entre les deux conditions en PIM et en POgt. Nous émettions l'hypothèse d'une position postérieure du CPP par l'augmentation de la dimension verticale d'occlusion. Le tableau 3 montre que les valeurs stabilométriques restent proches après la mise en place de l'orthèse tant les YO que les YF. Le fait de modifier la position mandibulaire en augmentant la dimension verticale a une influence sur la position de la tête (Greenberg, et al., 1981; Preiskel, 1965). Les auteurs constatent qu'une extension crânio-vertébrale entraîne une modification de l'équilibre entre la tête et le cou favorisant une modification des réponses posturales. Nos résultats ne remettent en aucun cas en cause le rôle de la dimension d'occlusion sur le placement de la tête et l'équilibre entre la tête et le cou mais ils infirment son influence sur les paramètres stabilométriques retenus.

La troisième question posée s'attache aux effets du port d'une orthèse occlusale toutes les nuits durant 15 et 30 jours, sur les réponses posturales. Nous voulions savoir si le fait d'avoir porté cette orthèse ainsi, modifiait ces réponses. Un des rôles de l'orthèse occlusale est de placer les patients d'une position dents serrées, muscles contractés, en PIM à une position myorelaxée grâce au port d'une orthèse. Nous savons qu'avant traitement, les patients bruxomanes sont en permanence, bouche fermée, dents serrées et rarement en position de repos (Brocard, Laluque, \& Knellssen, 2007). Bien que nous observions une influence significative du port de l'orthèse durant 30 jours sur la position médio-latérale en PR les YO $(p<0,01)$ et pour la position antéro-postérieure les YF en PIM $(p<0,02)$, il apparait difficile de définir un effet significatif majeur du port de l'orthèse durant 15 ou 30 jours. Les deux groupes n'évoluent donc pas de manière significativement différente à court et à moyen terme et il n'apparait pas d'influence de la modification des rapports intermaxillaires sur la stabilité et sur la projection du CPP des personnes bruxomanes.

Plusieurs limites peuvent être évoquées et participent à l'absence de mise en évidence de différences significatives. La première réside dans la non-prise en compte de l'influence de la masse corporelle sur les réponses posturales. L'observation des caractéristiques de notre population montre une masse moyenne de $77,7 \mathrm{~kg}$ pour les sujets sains et de $66,8 \mathrm{~kg}$ pour les sujets bruxomanes. Or, de nombreux travaux mettent en évidence l'influence du poids sur l'altération de la stabilité posturale (Handrigan, Corbeil, Simoneau, \& Teasdale, 2010 ; Hue, et al., 2007; Teasdale, Hue, Simoneau, Tremblay, Marceau \& Marceau, 2007). L'absence de différence significative entre les groupes pourrait être en relation avec des sujets du groupe sain, plus lourds, favorisant des réponses posturales plus amplifiées. Cette variable nécessite d'être contrôlée avec un appariement sur la masse. La seconde est liée à la petite taille des groupes et leur hétérogénéité est manifeste. Le recours à des tests non-paramétriques et la possible erreur statistique de type 2 constituent des limites qui imposent l'extension à de plus larges échantillons lors d'études futures. Les caractéristiques d'âge des sujets bruxomanes (32,4 ans) et donc leur faible ancienneté dans la pathologie pourraient en partie, justifier de l'absence d'influence claire du bruxisme sur les réponses posturales. De plus, le port d'une orthèse sur une durée maximale de 30 jours, au sein du protocole, constitue peut-être une période trop courte pour entraîner des effets et incite à reformuler l'hypothèse d'un effet à long terme.

\section{Conclusion}

Cette étude préliminaire menée dans le champ de l'occlusion dentaire et du bruxisme n'a pas permis de valider les hypothèses émises. Nous n'observons pas d'effet du bruxisme sur les valeurs de réponses posturales traditionnelles. Bien que non significative, une tendance à l'augmentation du paramètre de surface et une projection plus antérieure du CPP chez les sujets bruxomanes sont observables. Le port d'une orthèse occlusale sur une durée de 15 à 30 jours ne semble pas induire de modification marquante des réponses posturales. Cette durée devrait être augmentée afin d'envisager une éventuelle réorganisation des capacités de stabilisation.

Une analyse électromyographique lors de taches de stabilisation constitue la principale perspective pour mettre en évidence certaines conséquences physiologiques. L'activité intense voire constante d'une partie de la musculature maxillaire et nucale pourrait constituer une source de fatigue qu'il nous semble intéressant d'objectiver.

\section{Bibliographie}

American Academy of Neurology. Assessment: posturography. (1993). Report of the Therapeutics and Technology Assessment Subcommitee of the American Academy of Neurology, 43, 1261-1264.

Bonnier, L. (1996). Interaction occlusion et système postural. Le chirurgien dentiste de France, 9-16. 
Bricot, B. (1996). La reprogrammation posturale globale. Montpellier : Sauramps médical.

Bricot, B., \& Gahery, Y. (1981). Effet de l'occlusion dentaire sur le système postural. http://perso.orange.fr/ posturologie/rapports/rapp1.htm.

Brocard, D., Laluque, J.F., \& Knellssen, C. (2007). La gestion du bruxisme. Quintessence international.

Burkett, L.N., \& Bernstein, A.K. (1982). Strength testing after jaw repositioning with a mandibular orthopaedic appliance. Physician Sports Medecine, 10 (2), 103.

Chapman, R.J. (1991). Occlusal contact variation with changes in head position. International Journal of Prosthodontics, 4 (4), 377-381.

Clark, G.T., Rugh, J.D., \& Handelman, S.L. (1980). Noctural masseter muscle activity and urinary catecholamine levels in bruxers. Journal of Dental Research, 10, 1571-1576.

De Nunzio, A.M., Nardone, \& A., Schieppati, M. (2005). Head stabilization on a continuously oscillating platform: the effect of a proprioceptive disturbance on the balancing strategy. Experimantal Brain Research, 165 (2), 261-272.

Gangloff, P., Louis, J.P., \& Perrin, P.P. (2000). Dental occlusion modifies gaze and posture stabilization in human subjects. Neuroscience Letters, 293 (3), 203-6.

Greenberg, M., Cohen, S.G., Spinger, P., Kotwick, J.E., \& Vergo, J.J. (1981). Mandibular position and upper body strength: a controlled clinical trial. Journal of American Dental Association, 103.

Gresty, M. (1987). Stability of the head: studies in normal subjects and in patients with labyrinthine disease, head tremor, and dystonia. Movement Disorders, 2 (3), 165185.

Handrigan, G.A., Corbeil, P., Simoneau, M, \& Teasdale N. (2010). Balance control is altered in obese individuals. Journal biomecahnics, 19 (2), 383-384.

Hartmann, F., \& Cucchi, G. (1993). Les voies nerveuses et les projections centrales trigéminales. In Hartmann, F., \& Cucchi, G. Les dysfonctions cranio-mandibulaires. Paris : Springer-Verlag, pp. 9-15.

Hue, O., Simoneau, M., Marcotte, J., Berrigan, F., Doré, J., Marceau, P., Tremblay, A., \& Teasdale, N. (2007). Body weight is a strong predictor of postural stability. Gait \& Posture, 26, 32-38.

Kibana, Y., Ishijima, T., \& Hirai, T. (2002). Occlusal support and head posture. Journal of Oral Rehabilitation, 29 (1), 58-63.

Laplanche, O. (1992). Apport de l'occlusodontie au sportif de haut niveau : tests expérimentaux. Compte-rendu des neuvièmes journées internationales du Collège National d'Occlusodontologie, 323-328.

Larson, T.D. (2012). The effect of occlusal forces on restorations. Northwest Dental, 91 (6), 25-35.

Lexique Collège National d'Occlusodontie. (2001). Quintessence International.
Mc Lean, L.F., Brennan, H.S., \& Friedman, M.G.F. (1973). Effects of changing body position on dental occlusion. Journal of Dental Research, 52, 1041-1045.

Milani, R.S., De Perière, D.D., Lapeyre, L., \& Pourreyron, L. (2000). Relationship between dental occlusion and posture. Cranio, 18 (2), 127-134.

Norré, M.E. (1995). Posturography: head stabilisation compared with platform recording. Application in vestibular disorders. Acta Otolaryngoly Supplement, 520, 434-436.

Perinetti, G. (2006). Dental occlusion and body posture: no detectable correlation. Gait \&S Posture, 24 (2), 165-168.

Preiskel, H.W. (1965). Some observations on the postural position of the mandibule. Journal of Prosthetic Dentistry, 15 (4), 625-633.

Rey, J.P., Chossegros, C., El Zoghby, A., Carlier, J.F., Perez, C., \& Orthlieb, J.D. (2009). Le point sur les gouttières occlusales. Revue Odonto Stomatologie, 38, 1-13.

Rozenweig, D. (1994). Algies et dysfonctionnements de l'appareil manducateur. Éditions CDP.

Serviere, F. (1989). L'examen postural en occlusodontie quotidienne. Les Cahiers de Prothèse, 65, 37-42.

Solberg, W.K., Clark, G.T., \& Rugh, J.D. (1975). Nocturnal electromyographic evaluation of bruxism patients undergoing short term splint therapy. Journal of Oral Rehabilitation, 2, 215-223.

Stenger, J.M., Ricketts, J., Lawton, E.A., \& Wright, J.M. (1978). Muscular strength correlated to jaw posture and the temporo-mandibular joint: examination of a professional football population. New York State Dental Journal, 1272 .

Tardieu, C., Dumitrescu, M., Giraudeau, A., Blanc, J.L., Cheynet, F., \& Borel, L. (2009). Dental occlusion and postural control in adults. Neuroscience Letters, 30 (2), $221-224$.

Teasdale, N., Hue, O., Simoneau, M., Tremblay, A., Marceau, P., \& Marceau S. (2007). Predictors of weight loss in Parkinson's disease: is weight loss the chicken or the egg? Movement Disorders, 15 (3), 436-437.

Tripodakis, A.S., Smulow, J.B., Mehta, N.R., \& Clark, R.E. (1995). Clinical study of location and reproductibility of three mandibular positions in relation to body posture and muscle function. Journal of Prosthetic Dentistry, 73 (2), 190-198.

Unger, F., \& Brocard, D. (1996). Rôle des gouttières occlusales dans le traitement des désordres temporo-mandibulaires. Réalités cliniques, 7 (2), 219-228.

Urbanowicz, M. (1991). Alteration of vertical dimension and its effect on head and neck posture. Journal of Craniomandibular Practice, 9 (2), 174-179.

Vergo, J.J., Kotwick, J.E., Cohen S.G., \& Greenberg, M. (1981). The effect of orthopaedic intraoral mandibular appliance on upper body strength. Medecine Science Sports, 13 (2), 115. 